\title{
Bound on the multiplicity of almost complete intersections
}

\author{
Bahman Engheta \\ Department of Mathematics, University of California, Riverside, CA 92521 \\ E-mail address: engheta@math.ucr.edu
}

\begin{abstract}
Let $R$ be a polynomial ring over a field of characteristic zero and let $I \subset R$ be a graded ideal of height $N$ which is minimally generated by $N+1$ homogeneous polynomials. If $I=\left(f_{1}, \ldots, f_{N+1}\right)$ where $f_{i}$ has degree $d_{i}$ and $\left(f_{1}, \ldots, f_{N}\right)$ has height $N$, then the multiplicity of $R / I$ is bounded above by $\prod_{i=1}^{N} d_{i}-\max \left\{1, \sum_{i=1}^{N}\left(d_{i}-1\right)-\left(d_{N+1}-1\right)\right\}$.
\end{abstract}

Keywords: almost complete intersection, multiplicity, core

\section{Introduction}

Let $R=k\left[X_{1}, \ldots, X_{n}\right]$ where $k$ is a field of characteristic zero and let $f_{1}, \ldots, f_{N}$ be forms in $R$ of degrees $d_{1}, \ldots, d_{N}$, respectively, which generate an ideal of height $N$. That is, $f_{1}, \ldots, f_{N}$ form a regular sequence and they generate a complete intersection ideal with multiplicity $\prod_{i=1}^{N} d_{i}$. Let $f_{N+1}$ be yet another form, of degree $d_{N+1}$, which is a zero-divisor on $R /\left(f_{1}, \ldots, f_{N}\right)$, that is, $\left(f_{1}, \ldots, f_{N}\right) \subsetneq\left(f_{1}, \ldots, f_{N}\right): f_{N+1} \subsetneq R$. Herein the ideal $\left(f_{1}, \ldots, f_{N+1}\right)$ is referred to as an almost complete intersection. Throughout this article, let $I=\left(f_{1}, \ldots, f_{N+1}\right)$ and $\underline{f}=f_{1}, \ldots, f_{N}$. We will abuse the notation $\underline{f}$ to also denote the ideal generated by this sequence.

It can be shown, as one would expect, that the multiplicity of $R / I$ is strictly less than that of $R / f$. This note is aimed at making this fact more precise and exhibiting a bound for the multiplicity of $R / I$ in terms of the degrees $d_{1}, \ldots, d_{N+1}$ of the minimal generators of $I$. Our approach leads quite naturally to the notion of the core of an ideal, which was first alluded to by Rees and Sally in [RS] and treated more explicitly by Huneke and Swanson in [HS, and which has been the subject of growing interest in recent years. 
The core of an ideal. Recall that given two ideals $B \subseteq A, B$ is said to be a reduction of $A$ if $A^{t+1}=B A^{t}$ for some non-negative integer $t$, and consequently for all integers greater than $t$. (Equivalently, a reduction of $A$ is a subideal of $A$ with the same integral closure as $A$.) The least such integer is called the reduction number of $A$ with respect to $B$. A reduction $B$ of $A$ is called minimal if no ideal properly contained in $B$ is a reduction of $A$, that is, if it is minimal with respect to inclusion. As minimal reductions are not unique, one is led to consider the intersection of all minimal reductions: the core of an ideal $A$, denoted $\operatorname{core}(A)$, is defined as the intersection of all reductions (equivalently, minimal reductions) of $A$.

We first take advantage of the well-understood structure of $R / \underline{f}$ and express its multiplicity $\prod_{i=1}^{N} d_{i}$ as the length of $R /(\underline{f}, \underline{\ell})$, where $\underline{\ell}=\ell_{1}, \ldots, \ell_{n-N}$ is a sequence of $n-N$ general linear forms. Our focus will then be to determine when, and by how much, the additional generator $f_{N+1}$ further reduces the length of $R /(\underline{f}, \underline{\ell})$ to that of $R /(I, \underline{\ell})$. Finally, as the multiplicity of $R / I$ is no greater than the length of $R /(I, \underline{\ell})$, we obtain

$$
e(R / I) \leqslant \lambda(R /(I, \underline{\ell})) \lesseqgtr \lambda(R /(\underline{f}, \underline{\ell}))=e(R / \underline{f}),
$$

where $\lambda($ ) and $e($ ) denote length and multiplicity, respectively. More concretely, we prove the following

Theorem 1. Let $R$ be a polynomial ring over a field of characteristic 0 . If $I \subset R$ is an almost complete intersection minimally generated by $f_{1}, \ldots, f_{N+1}$ such that $f_{1}, \ldots, f_{N}$ form a regular sequence, then the multiplicity of $R / I$ is at most

$$
\prod_{i=1}^{N} d_{i}-\max \left\{1, \sum_{i=1}^{N}\left(d_{i}-1\right)-\left(d_{N+1}-1\right)\right\},
$$

where $d_{i}=\operatorname{deg}\left(f_{i}\right)$ for $i=1 \ldots N$.

Let $\bar{R}:=R / f$ and let ${ }^{-}$denote the residue class in $\bar{R}$. Our argument is based on the well-known fact (see $\overline{\mathrm{NR}}$ ) that in $\bar{R}$ any choice of $n-N$ general linear forms $\underline{\ell}$ generates a graded minimal reduction of the homogeneous maximal ideal $\overline{\mathfrak{m}}=\left(\bar{X}_{1}, \ldots, \bar{X}_{n}\right)$, and all graded minimal reductions of $\overline{\mathfrak{m}}$ are of this form.

Note that the strict inequality in (10) holds if and only if $f_{N+1} \notin(\underline{f}, \underline{\ell})$. Thus, to establish this inequality it suffices to show that the image of $f_{N+1}$ in $\bar{R}$ is not contained in some graded minimal reduction of $\overline{\mathfrak{m}}$. To this end, we appeal to a result of Corso, Polini, and Ulrich [CPU1, Theorem 4.5] which implies that in our setting core $(\overline{\mathfrak{m}})$ is in fact the intersection of the graded minimal reductions of $\overline{\mathfrak{m}}$, and we show that $\bar{f}_{N+1} \notin \operatorname{core}(\overline{\mathfrak{m}})$. To compute core $(\overline{\mathfrak{m}})$ we avail ourselves of a formula that was conjectured by Corso, Polini, and Ulrich in [CPU2, and was proved independently by Huneke and Trung [HT, Theorem 3.7] and Polini and Ulrich [PU, Theorem 4.5]. 


\subsection{Preliminaries}

We recall that given a sequence $\underline{\ell}=\ell_{1}, \ldots, \ell_{n-N}$ of general linear forms, the multiplicity of $R / I$ is bounded above by the length of $R /(I, \underline{\ell})$. Indeed, as $I$ has height $N$, the elements $\underline{\ell}$ constitute a system of parameters of $R / I$. The multiplicity of $R / I$ can be obtained as the Euler characteristic of $R / I$ with respect to $\underline{\ell}$

$$
\begin{aligned}
\chi(\underline{\ell}, R / I) & =\sum_{i \geqslant 0}(-1)^{i} \lambda\left(H_{i}(\underline{\ell}, R / I)\right) \\
& =\sum_{i \geqslant 0}(-1)^{i} \lambda\left(\operatorname{Tor}_{i}^{R}(R / \underline{\ell}, R / I)\right),
\end{aligned}
$$

where $H_{\bullet}(\underline{\ell}, R / I)$ denotes the Koszul homology of $\underline{\ell}$ with coefficients in $R / I$. If we further consider the first partial Euler characteristic

$$
\chi_{1}(\underline{\ell}, R / I)=\sum_{i \geqslant 1}(-1)^{i-1} \lambda\left(H_{i}(\underline{\ell}, R / I)\right),
$$

then we have $\chi(\underline{\ell}, R / I)=\lambda(R /(I, \underline{\ell}))-\chi_{1}(\underline{\ell}, R / I)$ and the following nonnegativity result yields $e(R / I) \leqslant \lambda(R /(I, \underline{\ell}))$, as desired.

Theorem (Serre $[\underline{S}])$. The first partial Euler characteristic $\chi_{1}(\underline{\ell}, R / I)$ is nonnegative, or equivalently, $\chi(\underline{\ell}, R / I) \leqslant \lambda(R /(I, \underline{\ell}))$.

Socle degree of complete intersections. Recall that in our setting $f$ is a regular sequence $f_{1}, \ldots, f_{N}$ with $\operatorname{deg}\left(f_{i}\right)=d_{i}$ and $\underline{\ell}=\ell_{1}, \ldots, \ell_{n-N}$ is a sequence of general linear forms. Next we point out that $R /(\underline{f}, \underline{\ell})$ has a pure socle generated in degree $\sum_{i=1}^{N}\left(d_{i}-1\right)$. To see this, we compute $\operatorname{Tor}_{n}^{R}(R /(\underline{f}, \underline{\ell}), k)$ twice. On the one hand, resolving $k$ via the Koszul complex on $X_{1}, \ldots, X_{n}$ and tensoring with $R /(\underline{f}, \underline{\ell})$ yields

$$
\operatorname{Tor}_{n}^{R}(R /(\underline{f}, \underline{\ell}), k) \cong \frac{(\underline{f}, \underline{\ell}): \mathfrak{m}}{(\underline{f}, \underline{\ell})}(-n) .
$$

On the other hand, resolving $R /(\underline{f}, \underline{\ell})$ via the Koszul complex on $\underline{f}, \underline{\ell}$ and tensoring with $R / \mathfrak{m}$ we have

$$
\operatorname{Tor}_{n}^{R}(R /(\underline{f}, \underline{\ell}), k) \cong k\left(-\left(n-N+\sum_{i=1}^{N} d_{i}\right)\right)
$$

Hence $\operatorname{socle}(R /(\underline{f}, \underline{\ell})) \cong k\left(-\left(\sum_{i=1}^{N} d_{i}-N\right)\right)$, as claimed. Also note that $\lambda(R /(\underline{f}, \underline{\ell}))=e(\bar{R} / \underline{f})=\prod_{i=1}^{N} d_{i}$.

Remark 2. For brevity set $r:=\sum_{i=1}^{N}\left(d_{i}-1\right)$. It follows that $\mathfrak{m}^{r+1} \subseteq(\underline{f}, \underline{\ell})$, or equivalently, $\mathfrak{m}^{r+1} \equiv \underline{\ell} \mathfrak{m}^{r}$ modulo $\underline{f}$. Thus, in $\bar{R}=R / \underline{f}$ the minimal reduction $\underline{\ell}$ of $\mathfrak{m}$ has reduction number $\leqslant r$. We also note that $\overline{\mathfrak{m}} \subset \bar{R}$ is an equimultiple ideal, that is, its height equals its analytic spread $n-N$. 


\section{Computation of the core}

The following theorem provides a formula for the core of an equimultiple ideal in a Cohen-Macaulay local ring.

Theorem 3 (Huneke-Trung [HT, 3.7], Polini-Ulrich [PU, 4.5]). Let $S$ be a Cohen-Macaulay local ring with residue field of characteristic 0 . Let $A$ be an equimultiple ideal of $S$ and let $B \subseteq A$ be a minimal reduction of $A$ with reduction number $r$. Then $\operatorname{core}(A)=B^{r+1}: A^{r}$.

Note that if $A$ is an equimultiple ideal, then its minimal reduction $B$ is generated by a regular sequence and it is easily seen that $B^{r+1}: A^{r}=B^{t+1}: A^{t}$ for all $t \geqslant r$. In PUV, Proposition 2.1] it is further shown that forming the core of zero-dimensional ideals commutes with localization. Thus, the colon formula of Theorem 3 may be applied in our setting (see also [PUV, Theorem 2.3]) and it yields

$$
\operatorname{core}(\overline{\mathfrak{m}})=\frac{\left(\underline{\ell}^{r+1}, \underline{f}\right): \mathfrak{m}^{r}}{\underline{f}},
$$

where $r=\sum_{i=1}^{N}\left(d_{i}-1\right)$ as set previously. Using (2) and minimal free resolutions, we now describe core $(\overline{\mathfrak{m}})$ more precisely with the following

Lemma 4. Let $R$ be a polynomial ring over a field of characteristic zero and let $\underline{f} \subset R$ be an ideal generated by a regular sequence of $N$ forms of degrees $d_{1}, \ldots, d_{N}$. Then core $(\overline{\mathfrak{m}})=\overline{\mathfrak{m}}^{r+1}$ with $r=\sum_{i=1}^{N}\left(d_{i}-1\right)$, where ${ }^{-}$denotes the residue class in $R / \underline{f}$.

Proof. Say $R=k\left[X_{1}, \ldots, X_{n}\right]$ and $\underline{f}$ is generated by the regular sequence $f_{1}, \ldots, f_{N}$ with $\operatorname{deg}\left(f_{i}\right)=d_{i}$. Set $\bar{R}:=R / \underline{f}$ and let $\underline{\ell}=\ell_{1}, \ldots, \ell_{n-N}$ be a sequence of general linear forms in $R$. By Remark 2 , the image of $\underline{\ell}$ in $\bar{R}$ generates a minimal reduction of $\overline{\mathfrak{m}}$ with reduction number $\leqslant r$ and by (2), $\operatorname{core}(\overline{\mathfrak{m}})=\frac{\left(\underline{\ell}^{r+1}, \underline{f}\right): \mathfrak{m}^{r}}{\underline{f}}$. To compute this colon, we first resolve $R /\left(\underline{\ell}^{r+1}, \underline{f}\right)$ to determine its socle degree. Let

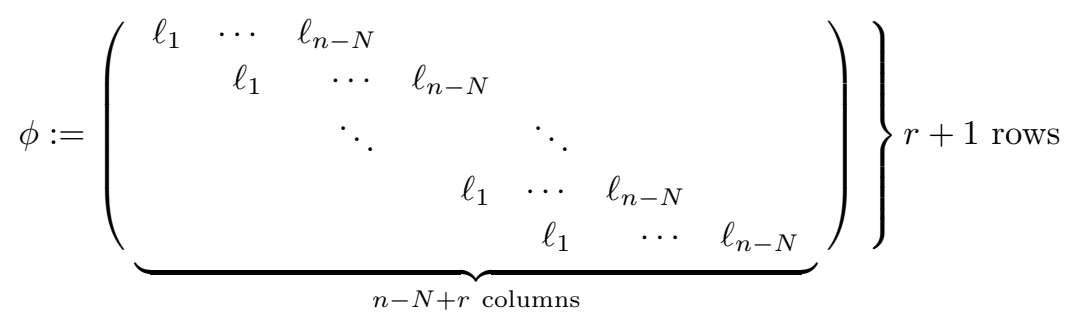

and note that the $(r+1) \times(r+1)$ minors of $\phi$ generate the ideal $\underline{\ell}^{r+1}$. Recall that $R / \underline{\ell}^{r+1}$ is perfect of grade $n-N$ and is minimally resolved by the Eagon- 
Northcott complex - see [EN]:

$$
\begin{aligned}
\mathbb{E N}(\phi): & 0 \rightarrow R^{b_{n-N}}(-(n-N+r)) \stackrel{d_{n-N}}{\longrightarrow} \cdots \\
& \cdots \rightarrow R^{b_{2}}(-(r+2)) \stackrel{d_{2}}{\longrightarrow} R^{b_{1}}(-(r+1)) \stackrel{\wedge^{r+1} \phi}{\longrightarrow} R \rightarrow R / \underline{\ell}^{r+1} \rightarrow 0 .
\end{aligned}
$$

On the other hand, $R / \underline{f}$ is minimally resolved by the Koszul complex:

$$
\mathbb{K}(\underline{f}): \quad 0 \rightarrow R\left(-\sum_{i=1}^{N} d_{i}\right) \rightarrow \cdots \rightarrow \bigoplus_{i=1}^{N} R\left(-d_{i}\right) \rightarrow R \rightarrow R / \underline{f} \rightarrow 0 .
$$

We consider the tensor product of the above complexes

$$
\begin{aligned}
\mathbb{E N}(\phi) \otimes \mathbb{K}(\underline{f}): \quad 0 \rightarrow R^{b_{n-N}}\left(-\left(n-N+r+\sum_{i=1}^{N} d_{i}\right)\right) \rightarrow \cdots \\
\left.\cdots \rightarrow R^{b_{1}}(-(r+1)) \oplus \bigoplus_{i=1}^{N} R\left(-d_{i}\right) \rightarrow R \rightarrow R / \underline{\ell}^{r+1}, \underline{f}\right) \rightarrow 0,
\end{aligned}
$$

and recall that its $i$-th homology is isomorphic to $\operatorname{Tor}_{i}^{R}\left(R / \underline{\ell}^{r+1}, R / \underline{f}\right)$ for $i \geqslant 0-$ see $[\mathrm{R}$, Theorem 11.21]. As $\underline{\ell}$ is generated by a regular sequence and $\underline{f}$ is a regular sequence modulo $\underline{\ell}$, it is also a regular sequence modulo $\underline{\ell}^{r+1}$ and all higher Tor vanish. Thus, $\mathbb{E N}(\phi) \otimes \mathbb{K}(\underline{f})$ is in fact a free resolution of $R /\left(\underline{\ell}^{r+1}, \underline{f}\right)$ of length $n$ in which the $n$-th module has a twist of $-\left(n+r+\sum_{i=1}^{N}\left(d_{i}-1\right)\right)=-(n+2 r)$. It now follows from

$$
\operatorname{Tor}_{n}^{R}\left(R /\left(\underline{r}^{r+1}, \underline{f}\right), k\right) \cong k^{b_{n-N}}(-(n+2 r)) \cong \frac{\left(\underline{\ell}^{r+1}, \underline{f}\right): \mathfrak{m}}{\left(\underline{\ell}^{r+1}, \underline{f}\right)}(-n) .
$$

that $R /\left(\underline{\ell}^{r+1}, \underline{f}\right)$ has socle isomorphic to $k^{b_{n-N}}(-2 r)$.

To prove our claim, let $x \in R$ such that $\bar{x} \in \operatorname{core}(\overline{\mathfrak{m}})$. As core $(\overline{\mathfrak{m}})=$ $\frac{\left(\underline{r}^{r+1}, \underline{f}\right): \mathfrak{m}^{r}}{\underline{f}}$, we have $x \mathfrak{m}^{r} \subseteq\left(\underline{r}^{r+1}, \underline{f}\right)$ and consequently $x \mathfrak{m}^{r-1}$ is contained in the socle of $R /\left(\underline{\ell}^{r+1}, f\right)$. This socle is generated in degree $2 r$, as shown above. Thus, $\operatorname{deg} x \geqslant r+1$ and core $(\overline{\mathfrak{m}}) \subseteq \overline{\mathfrak{m}}^{r+1}$. The reverse inclusion is clear, as $\mathfrak{m}^{2 r+1} \subseteq\left(\underline{\ell}^{r+1}, \underline{f}\right)$.

We are now ready to prove Theorem 1 .

Proof of Theorem 1. As before, let ${ }^{-}$denote the residue class in $\bar{R}=R / \underline{f}$ and let $\mathfrak{m}$ be the homogeneous maximal ideal $\left(X_{1}, \ldots, X_{n}\right)$. By Lemma 4 , core $(\overline{\mathfrak{m}})$ is generated in degree $r+1$, where $r=\sum_{i=1}^{N}\left(d_{i}-1\right)$. So if $\operatorname{deg}\left(f_{N+1}\right)=d_{N+1} \leqslant r$, then $\bar{f}_{N+1} \notin \operatorname{core}(\overline{\mathfrak{m}})$, that is, $\bar{f}_{N+1}$ is not contained in some minimal reduction of $\overline{\mathfrak{m}}$. 
By [CPU1, Theorem 4.5], core $(\overline{\mathfrak{m}})$ is in fact the intersection of general minimal reductions of $\overline{\mathfrak{m}}$. As the generators of $\overline{\mathfrak{m}}$ are all in degree one, its general minimal reductions are precisely the ideals generated by $n-N$ general linear forms. Thus, we have established that $f_{N+1} \notin(\underline{f}, \underline{\ell})$ for some choice of general linear forms $\underline{\ell}=\ell_{1}, \ldots, \ell_{n-N}$ whenever $d_{N+1} \leqslant r$. A priori, this implies

$$
\operatorname{HF}\left(R /(I, \underline{\ell}), d_{N+1}\right)=\operatorname{HF}\left(R /(\underline{f}, \underline{\ell}), d_{N+1}\right)-1,
$$

where $\operatorname{HF}(M, \quad)$ denotes the Hilbert function of an $R$-module $M$. But as $R /(f, \underline{\ell})$ is Gorenstein with a 1-dimensional socle in degree $r$, we have in fact

$$
\operatorname{HF}(R /(I, \underline{\ell}), i) \leqslant \operatorname{HF}(R /(\underline{f}, \underline{\ell}), i)-1 \quad \text { for } \quad d_{N+1} \leqslant i \leqslant r
$$

and consequently

$$
\lambda(R /(I, \underline{\ell})) \leqslant \lambda(R /(\underline{f}, \underline{\ell}))-\left(r-d_{N+1}+1\right) .
$$

As shown in Section 1.1, $e(R / I) \leqslant \lambda(R /(I, \underline{\ell}))$ and we arrive at

$$
e(R / I) \leqslant \prod_{i=1}^{N} d_{i}-\sum_{i=1}^{N}\left(d_{i}-1\right)+\left(d_{N+1}-1\right)
$$

whenever $d_{N+1} \leqslant r$.

Finally, notice that the condition $d_{N+1} \leqslant r$ is equivalent to $\sum_{i=1}^{N}\left(d_{i}-1\right)-$ $\left(d_{N+1}-1\right) \geqslant 1$. If this condition is not satisfied, one may infer by elementary means (see [E, Lemma 8]) that $e(R / I) \leqslant e(R / \underline{f})-1$. This proves the inequality

$$
e(R / I) \leqslant \prod_{i=1}^{N} d_{i}-\max \left\{1, \sum_{i=1}^{N}\left(d_{i}-1\right)-\left(d_{N+1}-1\right)\right\} .
$$

\section{Acknowledgments}

I thank Craig Huneke, Claudia Polini, and Bernd Ulrich for valuable conversations.

\section{References}

[CPU1] A. Corso, C. Polini, and B. Ulrich, The structure of the core of ideals. Math. Ann. 321 (2001), no. 1, 89-105.

[CPU2] A. Corso, C. Polini, and B. Ulrich, Core and residual intersections of ideals. Trans. Amer. Math. Soc. 354 (2002), 2579-2594. 
[EN] J.A. Eagon and D.G. Northcott, Ideals defined by matrices and a certain complex associated with them. Proc. Roy. Soc. Ser. A 269 (1962), 188204.

[E] B. Engheta, On the projective dimension and the unmixed part of three cubics. J. Algebra (2007), doi:10.1016/j.jalgebra.2006.11.018

[HS] C. Huneke and I. Swanson, Cores of ideals in 2-dimensional regular local rings. Michigan Math. J. 42 (1995), no. 1, 193-208.

[HT] C. Huneke and N.V. Trung, On the core of ideals. Compos. Math. 141 (2005), no. 1, 1-18.

[NR] D.G. Northcott and D. Rees, Reductions of ideals in local rings. Proc. Cambridge Philos. Soc. 50 (1954), 145-158.

[PU] C. Polini and B. Ulrich, A formula for the core of an ideal. Math. Ann. 331 (2005), no. 3, 487-503.

[PUV] C. Polini, B. Ulrich, and M. Vitulli, The core of zero-dimensional monomial ideals. Adv. Math. 211 (2007), no. 1, 72-93.

[RS] D. Rees and J.D. Sally, General elements and joint reductions. Michigan Math. J. 35 (1988), no. 2, 241-254.

[R] J.J. Rotman, An introduction to homological algebra. Pure and Applied Mathematics 85. Academic Press, 1979.

[S] J.-P. Serre, Local algebra. Springer Monogr. Math. Springer-Verlag, 2000 . 